\title{
Kommentar
}

Schweiz. Zschr. GanzheitsMedizin 17, 83-87 (2005). ㅇ Verlag für GanzheitsMedizin, Basel. www.ganzheitsmedizin.ch

\section{Reimar Banis}

mmer wieder werden mir als Entdecker der Psychosomatischen Energetik drei Fragen gestellt: «Wie haben Sie die homöopathischen Komplexe gefunden, mit denen seelische Konflikte geheilt werden?» und «Verträgt sich die Psychosomatische Energetik mit klassischer Homöopathie?» Die dritte Frage lautet: «Wie funktioniert die Psychosomatische Energetik (PSE) überhaupt und wie wirkt sie heilend?» Dass die Methode funktioniert, wird kaum mehr in Frage gestellt. Dass man mit homöopathischen Komplexmitteln seelische Konflikte dauerhaft auflösen und heilen kann, hat sich als Therapieform in kurzer Zeit in vielen naturheilkundlichen Praxen als Standard etabliert. Unklar ist jedoch für Viele, wie diese Heilung zustande kommt und mit welchen anderen Therapien sich die Methode verträgt. Das gilt insbesondere für Bachblüten und die klassische Homöopathie, aber auch für das Aufschwingen homöopathischer Substanzen auf bestimmte Träger.

Die Entdeckung der homöopathischen Komplexe der PSE war ein langwieriger Prozess, der sich über viele Jahre hinzog. Detailliert habe ich ihn im Lehrbuch der PSE beschrieben, was hier aus Platzgründen nicht wiederholt werden kann. Bei der Entdeckung der Komplexmittel wurde bald klar, dass zwei Faktoren entscheidend sind, um eine dauerhafte und optimale Konfliktheilung zu realisieren. Die Berücksichtigung dieser beiden Faktoren war auch die Grundlage bei der $\mathrm{Zu}$ sammenstellung der vierzig homöopathischen Emotionalmittel (Emvita ${ }^{\circledR}$ ). Der erste Faktor hat damit zu tun, dass seelische Konflikte aus einem Bündel unterschiedlicher Gefühlsfacetten be-

\section{Wie wirkt die Psychosomatische Energetik?}

\author{
Funktionsweise einer Therapie zur Heilung \\ seelischer Konflikte
}

stehen, die zwar unter einer Hauptüberschrift, dem übergeordneten Konfliktthema (Abb. 1), zusammengefasst werden können, aber tro $t$ zdem wegen der Vielschichtigkeit durch ein einziges Homöopathikum nur unzureichend wiedergegeben werden können.

\section{Seelische Konflikte mit zwei Qualitäten}

Wer sich sorgfältig beim Auftauchen einer starken Emotion analysiert, wird bestätigen, dass sich ein Hauptgefühl wie «Wut» mit ähnlichen Gefühlsvarianten zu einem bunten Potpourri zusammenmischt, also etwa mit Zorn, Hass, Ärger. Hinzu kommen parallel erlebte andersartige Gefühle, die mit «Wut» direkt nur wenig zu tun haben und sich dem Hauptgefühl beigesellen, wie Verzweiflung, Beschämung oder Trauer. Um deshalb einen seelis $\mathrm{c} h$ e $\mathrm{n}$ Konflikt wirklichkeitsgetreu wiedergeben zu können, muss ein dafür g eeignetes Komplexmittel unterschiedliche, aber dennoch verwandte Homöopathika enthalten, die das seelische Konfliktthema variieren und dadurch umfassend abbilden.

Die zweite Qualität, die einen seelischen Konflikt auszeichnet, betrifft seine Verankerung im Feinstofflichen. Ein Konflikt wirkt wie ein separatistisches Computerprogramm, das einem Computervirus oder inkompatiblem Programmteil vergleichbar die menschliche Software stört. Diese Software wird in der spirituellen Tradition als «Aura» oder «Ch,i, Od, Äther» bezeichnet und steuert als eine feldartige Hülle von Lebenskraft, die uns umgibt, sowohl unsere psychische wie körper- liche Verfassung. Damit hat dieses Energiefeld grundsätzlich eine psychosomatische Dynamik, und man kann soweit gehen zu behaupten, dass es keine Psychosomatik ohne die unmittelbare Mitwirkung der Lebenskraft gibt. Dadurch wird auch verständlich, weshalb jeder Konflikt Körper und Seele beeinflusst, so dass eine Konfliktbehandlung immer eine ganzheitliche Therapie sein wird.

Die eigentliche Ursache der meisten psychosomatischen Störungen beruht nach den Erkenntnissen der Psychoanalyse ebenso wie der modernen Traumaforschung auf unbewussten, in der Ve rgangenheit nicht vollständig verbeiteten Traumata (Konflikten). Um einen Konflikt besser zu verstehen, sind einige Anmerkungen notwendig. Der bereits angeführte Vergleich mit dem Computervirus erklärt das Subversive und Geheime, das jeden Konflikt auszeichnet. Zwar ahnen viele Menschen, das hinter ihren seelischen und körperlichen Krankheiten ein Konflikt verborgen sein könnte, aber er versteckt sich vor ihnen auf raffinierte Weise und benötigt Hilfsmittel, etwa die Testung mit der PSE, um ans Tageslicht geholt zu werden.

Konflikte können wegen dem Phänomen ihrer Verdrängung auch nur unzureichend mit normalem Bewusstsein erkannt werden. Dazu gehört auch das Befragen im Rahmen der klassischen Homöopathie (Repertorisieren). Das wird gelegentlich von Homöopathen geleugnet, weil sie vorgeben, unbewusstes Material wie Träume bei der Mittelfindung zu berücksichtigen. Doch die Praxiserfahrung zeigt eindeutig, dass dem in der Regel nicht so ist, denn ein homöopathisches Einzelmit- 


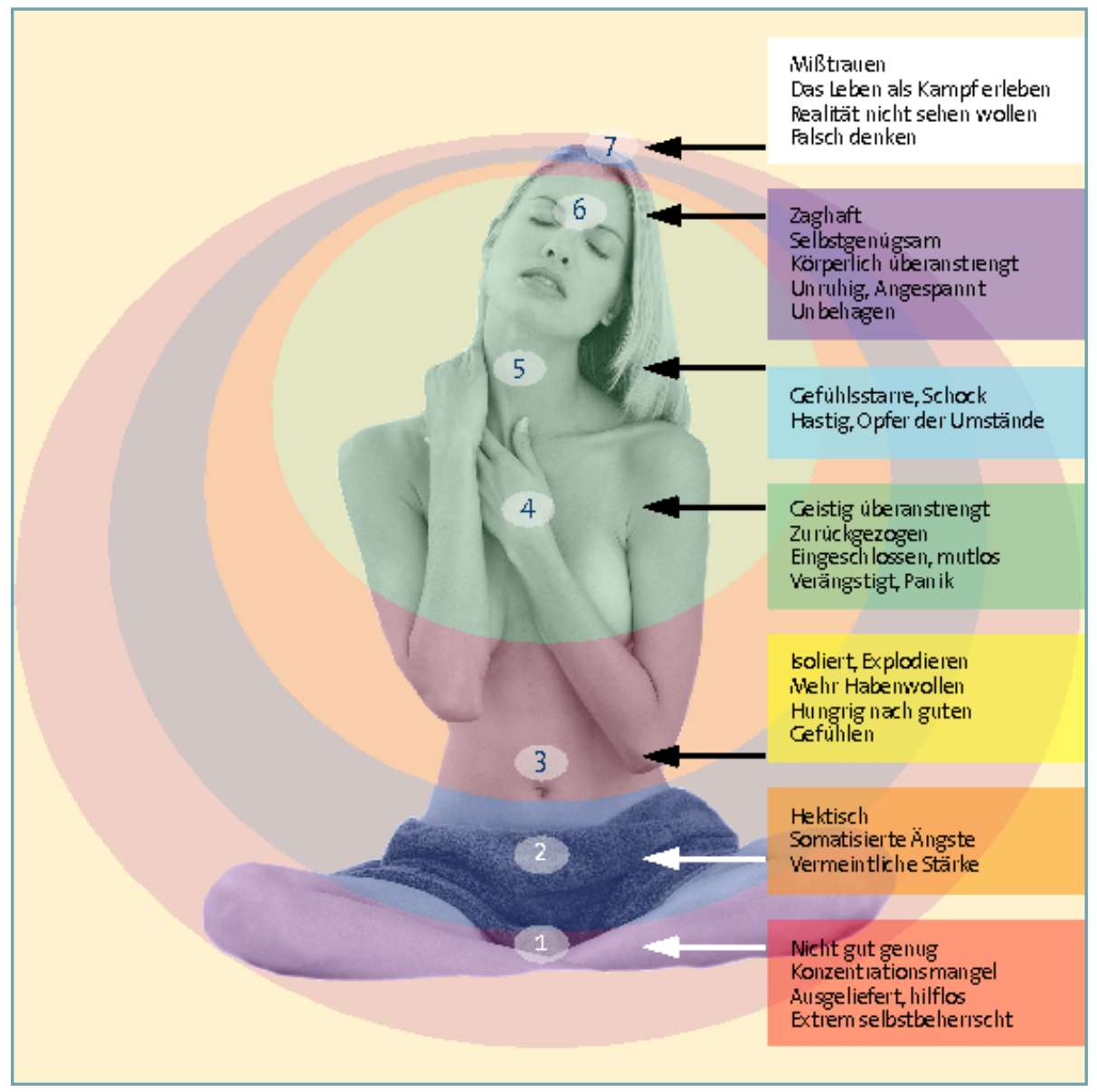

Abb. 1. Kurzübersicht der 28 Konfliktthemen

tel, das einem Patient zuvor vom klassischen Homöopathen verordnet worden ist, hat meist nichts mit dem Konfliktthema der PSE zu tun. Die klassische Homöopathie scheint mir bereits an der ersten Hürde $\mathrm{zu}$ scheitern, nämlich dem Unbewussten des Konflikts und seiner Tendenz, sich zu verbergen und verdrängt zu werden.

\section{Homöopathie: Feinstoffliche Ebenen und Emotionalmittel}

Um auf das Feinstoffliche zurückzukommen, hat die Lebenskraft des Menschen nach alter spiritueller Tradition eine unterschiedliche Schichtung in Form von vier Aurahüllen (vital, emotional, mental und kausal). Nun besitzt der Konflikt nach den Erkenntnissen der PSE ebenso alle vier Aurahüllen, wie sie der Mensch als sein Konfliktträger aufweist (siehe Abbildung 2). Da aus der Sicht der Homöopathie jede höhere feinstoffliche Ebene resonanzmässig mit einer höheren Potenz verknüpft ist, kann man einen Konflikt mit seinen unterschiedlichen AuraAnteilen logischerweise nicht mit einer einzigen homöopathischen Potenz behandeln, sondern benötigt unterschiedliche, teilweise extrem hohe Potenzen, um die verschiedenen Konfliktanteile, aus denen er sich zusammensetzt, resonanzmässig anzusprechen.

Die Emotionalmittel enthalten deshalb verschiedene Potenzen bis $\mathrm{zu}$ sehr hohen Potenzen wie der LM 18 und $\mathrm{C} 800$, die man für die höchsten Aura-Ebenen benötigt. Selbst wenn manchem klassischen Homöopathen die Zusammensetzung ungewöhnlich anmutet, verlangen es die Gesetzmässigkeiten des Konflikts dennoch, auch wenn das zunächst gegen orthodoxe homöopathische Regeln verstösst. Denn man muss so verfahren, damit alle feinstofflichen Schwingungsebenen erfasst werden. Die Erfahrung zeigt, dass dieser Ansatz zum Erfolg führt, und zwar nur er alleine, denn nur das perfekt passende Schwingungsmittel heilt den Konflikt vollständig, vergleichbar einem Tresor, der auch nur dann aufspringt, wenn man zuvor die richtige Tastenkombination eingegeben hat.

Zusätzlich enthalten die Emotionalmittel einen weiteren homöopathischen Kunstgriff, der aus einem Potenzakkord besteht. Die Potenz einer D 21 ähnelt nach meinen Forschungen schwingungsmässig der Urtinktur und enthält latent alle anderen Potenzen, was zu einer besonders breiten Wirkung führt. Bereits HANS HEINRICH RECKEWEG als der Entdecker der Homotoxikologie hat erkannt, dass Potenzakkorde wie D 4, D 6, D 12, D 30 eine viel breitere Wirkung haben als ein Einzelmittel. Weiter war REINHARD VOLL bei der Elektroakupunkturtestung aufgefallen, dass beim gleichen Patienten an unterschiedlichen Tagen verschiedene Potenzen des gleichen Homöopathikums ansprechen können. Der Potenzakkord als D 21 im jeweiligen Emotionalmittel stellt ständig alle benötigten Potenzen bereit. Es versteht sich von selbst, dass das Einzelmittel in D 21 wegen dieser breiten Wirkung zugleich das eigentliche homöopathische Hauptmittel des jeweiligen Komplexes darstellt. Es wirkt gezielt auf das Hauptgefühl, das einen Konflikt prägt (Abb. 1).

Die Emotionalmittel zu einem Komplex zusamenzusetzen war danach eine Fleissarbeit. Er bestand aus einer Mischung von Repertorisieren (Fachbegriff für das Heraussuchen aus homöopathischen Nachschlagewerken) und aus wiederholter Energietestung an Patienten. Die ersten therapeutischen Erfahrungen mit den Emotionalmitteln waren erstaunlich, denn es fehlten trotz der langen Anwendungsdauer von Monaten und der Gabe höchster Potenzen irgendwelche Zeichen eines homöopathischen Arzneimittelbildes. Dieses pflegt für gewöhnlich nach einiger Zeit aufzutauchen, wenn hochpotenzierte Homöopathika längere Zeit gegeben werden. Es daue rte einige Zeit, erford e rte einige Überlegung und wiederholte Energietestung, bis klar wurde, dass die Emotionalmittel vollständig vom Konflikt absorbiert werden, vergleichbar einem 


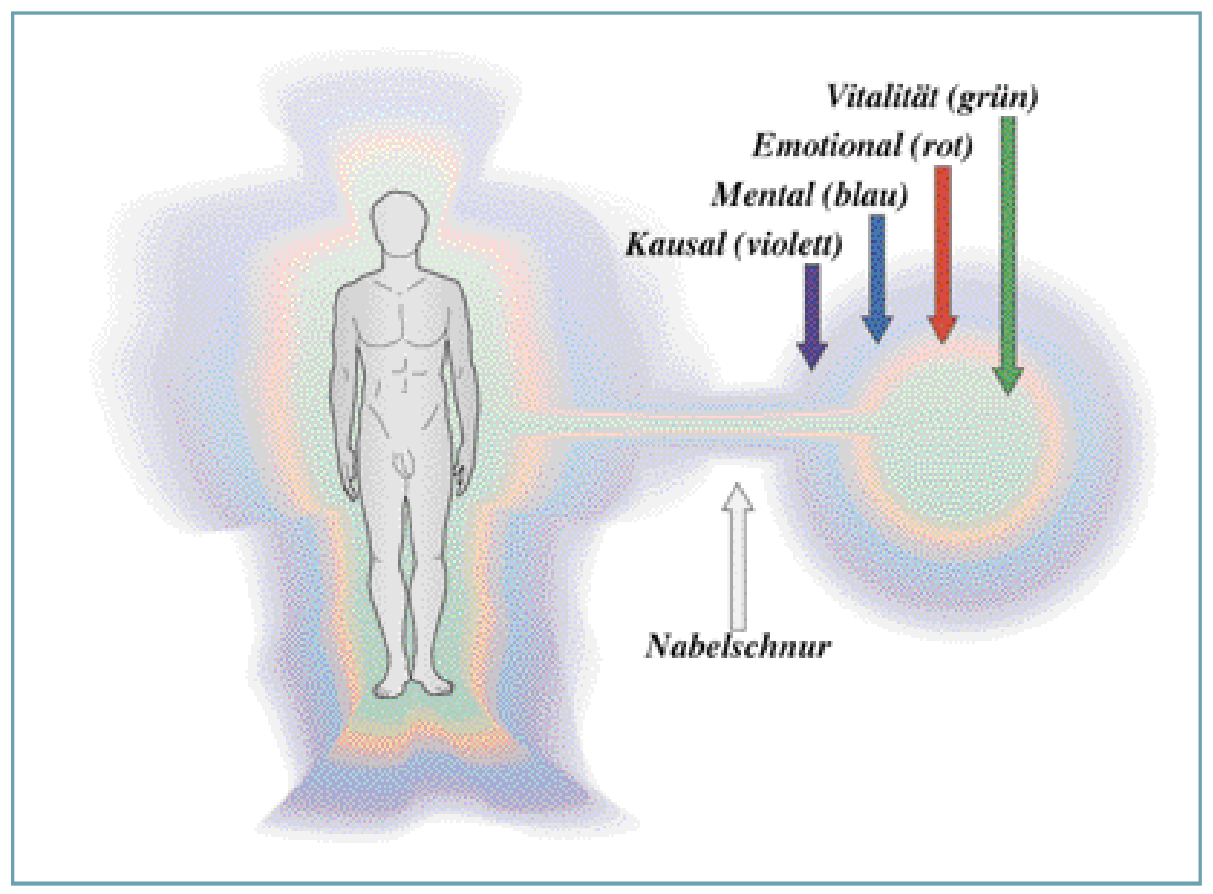

Abb. 2. Die vier Energiefelder eines Konflikts sowie die Nabelschnur, die inn mit dem Kraftfeld des Konfliktträgers verbindet.

schwarzen Loch, das Umgebungslicht aufsaugt. Daher kann das Emotionalmittel keine systemischen Wirkungen beim Patienten hervorrufen, so dass auch kein Arzneimittelbild entsteht. Man kann die Emotionalmittel deshalb im strengen Sinn nicht als Homöopathika bezeichnen, obwohl es sich um homöopathische Komplexe handelt, weil sie bei ihrer Anwendung nicht vollständig den Regeln Hahnemanns folgen.

Gängige Einzelmittel-Homöopathika dagegen wirken nicht auf den Konflikt, oder wenn sie das tun, dann nur sehr partiell und nicht tiefgreifend genug. Doch sie wirken auf einer anderen Ebene, etwa als Similimum, indem sie auf die gesamte Lebenskraft des Patienten einwirken. Diese gesamte Lebenskraft (in Form des Feldes, das den Patient umgibt, siehe Abbildung 2) wird durch den Konflikt auf indirekte Weise beeinträchtigt und gestört. Natürlich ist es sinnvoller, therapeutisch an der primären Störquelle anzusetzen, nämlich am Konflikt, doch das Harmonisieren der gesamten Lebenskraft des Patienten durch EinzelmittelHomöopathika hat ebenfalls seine Berechtigung. So spürt der Patient dadurch viel schneller einen Soforteffekt und wird gleichzeitig auf eine umfas- sende Weise harmonisiert. Man kann beide Therapien gut kombinieren, indem ein gut repertorisiertes Einzelmittel für schnelle, den Patienten zufriedenstellende Effekte sorgt, während das Emotionalmittel in einem sich über mehrere Monate hinziehenden Prozess den Konflikt abschmilzt. Zusammenfassend kann ich sagen, dass Bedenken von Seiten klassischer Homöopathen bezüglich der Parallelanwendung der PSE völlig fehl am Platze sind, man im Gegenteil sogar sagen kann, dass sich beide Therapien hervorragend ergänzen.

Das gilt auch für Bachblüten, die in ähnlicher Weise wie homöopathische Einzelmittel einzustufen sind, allerdings wegen ihrer sehr feinen und hohen Schwingung mehr auf den Kausalbereich wirken. Gerade bei Bachblüten kann man klar erkennen, dass es sich wegen ihres nicht ursächlich den Konflikt heilenden Therapieansatzes um eine kurzfristig wirkende Therapie handelt, die wegen ihrer schwachen Wirkung dazu neigt, polypragmatisch $\mathrm{zu}$ werden, indem mehrere Blütenmittel kombiniert werden müssen. Die Kombination von Emotionalmitteln der PSE und Bachblüten wirkt dagegen hervorragend, indem die
Nachteile der langwierigen Konfliktlöschung der PSE durch den raschen Wirkungseintritt der Bachblüten abgemildert und für den Patienten subjektiv ausgeglichen werden.

\section{«Therapiebeschleuniger»}

Weiter möchte ich auf die häufig gestellte Frage zurückkommen, was alternativ zur PSE Konflikte abheilen lässt beziehungsweise, was die Therapie mit der PSE beschleunigt. Mittlerweile verfügen über hundert Therapeuten, die seit Jahren die PSE schwerpunktmässig einsetzen, über zahlreiche Erfahrungen mit anderen Therapien, sei es, dass bestimmte Therapeuten andere Verfahren parallel anwenden oder dass Patienten damit von anderen Therapeuten vorbehandelt worden sind. In allen Fällen besteht durch die Messung der Aura-Ladung des Patienten mit dem Reba ${ }^{\circledR}$-Testgerät die Möglichkeit, quantitativ zu messen, wie es einem Patienten vorher und nachher geht. Wenn ein Patient nach einer Therapie konstant eine stark reduzierte Vitalität hat, kann man eine Vorbehandlung mit einer bestimmten Methode sicher nicht als erfolgreich einstufen, insbesondere, wenn das immer wieder und nicht nur bei einem Patienten vorkommt. Überzeugend ist auch, wenn Therapeuten die PSE und eine bestimmte Methode abwechselnd anwenden und dadurch Vergleiche anstellen können, die zu den gleichen Ergebnissen gelangen.

Die Testung mit der PSE hat sich mehr und mehr als eine Art Lackmusprobe erwiesen, die ganz generell mithelfen kann, die Qualität einer beliebigen Heilmethode quantitativ zu messen. Dabei zeigte sich zur allgemeinen Überraschung aller Beteiligten, dass erstaunlich viele Therapieverfahren entweder nur als Plazebo wirken oder kurzfristige Initialeffekte haben, etwa viele Vitamine, Spurenelemente und zahlreiche OTC-Produkte. Auch viele andere Therapieverfahren sind längst nicht so erfolgreich, wie behauptet wird, insbesondere bei Langzeitbeobachtung. Ganz pauschal ist bei der Testung mit dem Reba ${ }^{\circledR}$ Testgerät beispielsweise unter zehn 
Naturheilmitteln, die ein bestimmter Patient als Dauermedikamente einnimmt, meist nur ein Mittel, das einen messbaren Effekt hat. Man kann also zusammenfassen, dass erstaunlich wenige Medikamente auf Dauer wirkliche Heilmittel sind, das heisst im Mittel weit unter $10 \%$.

Das Gleiche gilt für viele Therapien, die oft geringere Effekte haben, als von ihren Protagonisten behauptet wird. Viele Heilmethoden wirken darüber hinaus nicht kausal, indem zugrundeliegende Energieblockaden und sonstige Störfaktoren beseitigt werden, sondern dadurch, dass die gesamte Vitalität des Patienten einige Zeit angehoben und harmonisiert wird, wodurch er zwar zunächst eine Besserung verspürt, doch der Effekt nicht dauerhaft anhält. Das gilt insbesondere für das Aufschwingen von Homöopathika, ein Verfahren, das zur Konfliktauflösung vollkommen ungeeignet ist, da es Konflikte auf Dauer unbeeinflusst lässt, teilweise sie sogar vergrössert. Ein weiteres Problem einer ungenügenden Therapie betrifft das Entkoppeln von Konflikten, etwa durch die Psychokinesiologie (PK), wodurch häufig eine zwar zunächst scheinbare, aber leider nicht dauerhafte Heilung vorgetäuscht wird. Auch hier gilt, dass der gute Initialeffekt der PK durch die Konfliktlöschung mittels der PSE kombiniert werden sollte.

Positive Effekte auf die Abheilung von Konflikten werden von all solchen Psychotherapieverfahren berichtet, die den Konflikt zunächst initial kurz aktivieren, wobei das so schonend wie möglich geschehen sollte, um nicht alte Wunden aufzureissen und den Konflikt im ungünstigsten Fall sogar zu vergrössern. Nach der Aktivierung besteht deren hauptsächliches Ziel darin, das alte Trauma zu einem guten Ende zu bringen. Gewissermassen dürstet die Seele nach dem Trauma nach einem Happy End, das jetzt zwar künstlich im therapeutischen Setting nachgeholt wird, aber der verwundeten Seele trotzdem erlaubt, das traumatische Erlebnisse endgültig zu verarbeiten und damit erfolgreich abzuschliessen.

So berichtet der Hypnotherapeut JöRG MEYER von hervorragenden Er- gebnissen bei Suchtbehandlung, wenn das durch die PSE getestete Konfliktthema zu einem glücklichen Ende gebracht wird, wobei in Hypnose der Zustand der Suchtlosigkeit suggestiv mit Zufriedenheit, Freude und dergleichen gekoppelt wird und zugleich eine PSE-Therapie durchgeführt wird. In ähnlicher Weise berichtet die Kunsttherapeutin BETTINA EGGER von guten Erfolgen, wenn der Konflikt mit der PSE abgeschmolzen und gleichzeitig kunsttherapeutisch behandelt wird. Die Erfolge dieser Kombinationstherapie sind deutlich besser als die alleinige Kunsttherapie, wobei schon die Kunsttherapie alleine nach ihren Beobachtungen auf den Konflikt messbar abheilend wirkt. Bettina Egger lässt den Patient szenisch Bilder malen, die aus dem Unbewussten aufsteigen und Schnappschüssen ähneln, die wie im Film das Trauma nachstellen. Der Patient wird aufgefordert, sich neue Szenen vorzustellen und sie zu malen, die ein imaginiertes Happy End darstellen, wobei das Malen des Bildes vom Unbewussten erstaunlicherweise als Realität gewertet wird, selbst wenn es sich nur um ein virtuelles Bild handelt.

In ähnlicher Weise wirkt auch das systemische Familienstellen, das letztlich auf ein harmonisches, alle Familienmitglieder versöhnendes Endbild abzielt. Familienstellen wirkt am besten, wenn es mit der PSE kombiniert wird, wie eine von uns durchgeführte kleine Studie an einigen wenigen Patienten gezeigt hat. Patienten mit dieser Kombination verloren ihre Konflikte dauerhaft, während nicht behandelte Patienten, die eine Einnahme der PSE-Tropfen ablehnten, eine unveränderte Konfliktgrösse gehabt haben. Da wir bei vielen Patienten trotz vorherigem Familienstellen nach wie vor grosse Konflikte finden, scheint dem eine allgemeingültige Bedeutung zuzukommen (Familienstellen heilt keine Konflikte, sondern aktiviert sie, das allerdings in erheblicher Weise).

\section{Wie wirkt die PSE?}

Abschliessend möchte ich kurz auf die Frage eingehen, wie die PSE überhaupt wirkt, denn vielen Therapeuten leuchtet es nicht unmittelbar ein, dass alleine die Gabe homöopathischer Tropfen solche weitreichenden Effekte haben soll, die in vielen Fällen sogar eine Psychotherapie ersetzen kann. Begleitend gegeben kann sie eine Psychotherapie wesentlich verkürzen und gleichzeitig verstärken, indem man schneller «auf den Punkt» kommt. Darüber hinaus wirkt die PSE während des Heilprozesses nicht nur auf die Seele, da oft erstaunliche körperliche Regenerationen erlebt werden. Der erste Grund für die tiefgreifende und ganzheitliche Wirkung der PSE besteht darin, dass Konflikte wie erwähnt unsere gesamte Befindlichkeit in seelisch-körperlicher Hinsicht stören. Das komplexe Schwingungsmuster des Konflikts wird durch das ähnliche Komplexhomöopathikum nach und nach gelöscht. Dadurch entfällt mehr und mehr der Störeinfluss des Konflikts, und das normalerweise harmonische Feld der Lebenskraft kann sich daraufhin neu reorganisieren und Schritt für Schritt erholen.

Parallel kommt es zu einer seelischen Neuordnung, die mit einer «Eigen-Psychotherapie» umschrieben werden kann. Damit entfällt das unbewusste Störprogramm des Konflikts, das mit einer Souffleuse im Theater verglichen werden kann, die in der Vergangenheit unbemerkt die Regie des Theaterstücks «Leben» mehr oder minder zu übernehmen versucht hat, während der Mensch als eigenverantwortlicher Lenker seines Lebens eingeschränkt war, im Extremfall sogar zur Marionette degradiert war. Natürlich handelt es sich bei diesem Störprogramm nicht nur um einen Prozess passiven Ausgeliefertseins, den man bei Ich-schwachen Individuen möglicherweise als Exkulpation unterstellen kann, sondern im Normalfall herrscht eine mehr oder minder deutliche Kumpanei von Konflikt und Konfliktträger.

Psychoanalytisch spricht man vom «Widerstand», wenn der Konfliktträger damit konfrontiert wird, seinen Eigenanteil am neurotischen Programm aufzugeben und dies jedoch nicht will, womit sein Eigenanteil deutlich wird. Diesen Widerstand erleben wir auch in der PSE, entweder in 
Form aller möglichen Ausreden, die Therapie vorschnell zu beenden, interessanterweise in Form systemischer Verstrickungen stellvertretend oft als Wunsch von Angehörigen vorgebracht (häufig bei Kindern), oder als körperliche Reaktionen, die Erstverschlimmerungen ähneln und oft mit Ausscheidungsreaktionen wie Durchfall, vermehrtem Wasserlassen, anfänglicher Verschlimmerung von Hautausschlägen und dergleichen einhergehen, so dass ein Therapieabbruch als geeignetes Mittel erscheint. Der Patient muss bereit sein, an diesem kritischen Punkt den Heilungsverlauf nicht vorschnell abzubrechen, gestärkt von dem Wunsch, unbedingt gesund werden zu wollen. Denn wer seine Heilung offen ablehnt und sogar boykottiert, dem kann auch die PSE nicht helfen, aber letztlich auch keine andere Therapie, die wie die PSE auf die Selbststeuerung des Individuums abzielt. Trotzdem ist die PSE keine Suggestiv- oder Placebobehandlung, da mir ein nicht unbedeutender Teil der Patienten später erzählt, anfänglich mit einer Hei- lung nicht gerechnet zu haben.

Während der PSE-Therapie beginnt bei vielen Patienten ein Prozess der seelischen Neuorientierung hin zu mehr Eigenverantwortung, zu mehr Lebendigkeit und Sinnesfreude, auch zu verbesserter Abgrenzung wie der umgekehrten Fähigkeit, sich vorbehaltloser anderen Menschen und neuen Erfahrungen zu öffnen. Natürlich entstehen solche Fähigkeiten und Zustände nicht von selbst, sondern müssen gewollt und zugelassen werden, ausserdem muss man sie aktiv trainieren, um darin Übung zu bekommen. All diese Vorgänge geschehen beim $G$ rossteil aller Menschen erstaunlicherweise ohne fremdes Zutun im Verlauf einer Eigentherapie, die gewissermassen selbststeuernd und selbstregulierend abläuft. Eine Psychotherapie ist deshalb im allgemeinen entbehrlich und nur dann bei der PSE sinnvoll, wenn entweder zu starke Neurotizismen mit entsprechenden Widerständen da sind, oder das Ich zu wenig Kraft für eine Eigentherapie aufbringen kann.

\section{Literatur}

Banis, Reimar: Durch Energieheilung zu neuem Leben. Verlag Via Nova Petersberg 2002

Banis, Reimar: Psychoenergetische Testung beim Familienstellen, Co'med 4/1999.

Banis, Reimar: Psychosomatische Energetik in der Allgemeinpraxis. Schweiz. Zschr. GanzheitsMedizin 2002;14(2):75-84.

Banis, Ulrike: Handbuch der Psychosomatischen Energetik. Co'med Verlag Hochheim 1999.

Banis, Ulrike: Naturheilkundliche Arbeit mit Kindern. Ermutigende Erfahrungen durch den Einsatz der Psychosomatischen Energetik. Schweiz. Zschr. GanzheitsMedizin 2002;14(7/8): 370-373.

Egger,Bettina: Vortrag bei der Medizinischen Woche Baden-Baden 2004

Klußmann, Rudolf: Psychosomatische Medizin. Springer Heidelberg 1998.

Meyer, Jörg: Psychosomatische Energetik als Voraussetzung für eine effektive Hypnosetherapie, in: Banis, R (Herausgeber): Lesebuch der Psychosomatischen Energetik (Band 1), Co'med 2004.

Reckeweg, Hans Heinrich: Homotoxikologie, Aurelia Verlag. Baden-Baden 1975.

Voll, Reinhard: Elektroakupunkturfibel. MLV Verlag Uelzen 1975.

\section{Anschrift des Autors:}

Dr. med. Reimar Banis

Grosmatt 3, CH-6052 Hergiswil

drbanis@hotmail.com 